\title{
Teste alfa de uma tecnologia gamificada para crianças e adolescentes em hemodiálise
}

\author{
Alfa test of a gamified technology for children and adolescents in hemodialysis \\ Prueba alfa de una tecnología gamificada para niños y adolescentes en hemodiálisis
}

Fernanda de Nazaré Almeida Costa $^{1}$ (1) Paulo Elias Gotardelo Audebert Delage ${ }^{2}$ (1) Mary Elizabeth de Santana ${ }^{3}$ (D) Marcia Helena Machado Nascimento ${ }^{3}$ (D) Elizabeth Teixeira ${ }^{4}$ (D)

1. Centro Universitário Mauricio de Nassau, Curso de Enfermagem. Belém, PA, Brasil. 2. Universidade do Estado do Pará, Departamento de Filosofia e Ciências Sociais. Belém, PA, Brasil.

3. Universidade do Estado do Pará, Departamento de Enfermagem Hospitalar. Belém, PA, Brasil.

4. Universidade do Estado do Amazonas, Curso de Enfermagem. Manaus, AM, Brasil.

Autor correspondente:

Fernanda de Nazaré Almeida Costa. E-mail: fepedrinho@yahoo.com.br.

Recebido em 04/02/2021.

Aprovado em 11/08/2021.

DOl:https://doi.org/10.1590/2177-9465-EAN-2020-0514

\section{RESUMO}

Objetivo: apresentar o teste alfa do protótipo de uma tecnologia gamificada, o Nefro Hero ${ }^{\circledR}$, com foco na Qualidade de Vida Relacionada à Saúde de pacientes pediátricos em tratamento hemodialítico. Método: pesquisa metodológica, avaliativa, por meio do teste alfa, com abordagem quantitativa, realizada com oito pacientes em tratamento hemodialítico com idades entre oito e 16 anos. Foi desenvolvida em quatro etapas: primeira aplicação do inventário de QVRS DISABKIDS ${ }^{\circledR}$; nove sessões de jogo com os pacientes, individualmente, durante as sessões de hemodiálise; segunda aplicação do inventário; duas sessões de brainstorming. Resultados: entre a primeira e a segunda aplicações do inventário, foram identificadas melhorias no domínio mental na QVRS dos pacientes, indicando o potencial da função primária da estratégia. Foram levantados pontos para ser revistos na tecnologia antes da etapa de validação. Conclusão e implicações para a Enfermagem: por ser um teste alfa, os resultados não podem ser generalizados, mas indicam o potencial da estratégia. $\mathrm{O}$ artigo apresenta uma etapa de avaliação anterior à de validação, fundamental ao desenvolvimento de tecnologias em formato de jogos e ainda pouco explorada.

Palavras-chave: qualidade de vida; diálise renal; jogos e brinquedos; criança; adolescente.

\section{ABSTRACT}

Objective: to present the alpha test of a prototype of a gamified technology, the Nefro Hero®, focused on the Health-Related Quality of Life of pediatric patients on hemodialysis treatment. Method: a methodological, evaluative research, through the alpha test, with quantitative approach, carried out with eight patients on hemodialysis treatment aged between eight and 16 years It was developed in four stages: first application of the DISABKIDS $®$ HRQoL inventory; nine game sessions with the patients, individually, during the hemodialysis sessions; second application of the inventory; two brainstorming sessions. Results: between the first and second applications of the inventory, improvements were identified in the mental domain in the patients' HRQoL, indicating the potential of the primary function of the strategy. Points to be reviewed in the technology before the validation stage were raised. Conclusion and Implications for Nursing: as it is an alpha test, the results cannot be generalized, but they indicate the potential of the strategy. The article presents an evaluation stage prior to validation, fundamental to the development of game-format technologies and still little explored.

Keyword: quality of life; kidney dialysis; games and toys; child, adolescent.

\section{RESUMEN}

Objetivo: presentar la prueba alfa del prototipo de tecnología gamificada, Nefro Hero®, con foco en la Calidad de Vida Relacionada con la Salud en pacientes pediátricos en hemodiálisis. Método: investigación metodológica, evaluativa, mediante la prueba alfa, con abordaje cuantitativo, realizada con ocho pacientes en hemodiálisis con edades comprendidas entre los ocho y los 16 años. Se desarrolló en cuatro etapas: primera aplicación del inventario HRQL DISABKIDS®; nueve sesiones de juego con pacientes individualmente durante las sesiones de hemodiálisis; segunda aplicación del inventario; dos sesiones de brainstorming. Resultados: entre la primera y la segunda aplicación del inventario, se identificaron mejoras en el dominio mental de la CVRS de los pacientes, indicando el potencial de la función primaria de la estrategia. Se plantearon puntos para ser revisados en la tecnología antes de la etapa de validación. Conclusión e implicaciones para Enfermería: al tratarse de una prueba alfa, los resultados no pueden generalizarse, pero indican el potencial de la estrategia. El artículo presenta una etapa de evaluación previa a la validación, fundamental para el desarrollo de tecnologías de formatos de juego y aún poco explorada.

Palabras clave: calidad de vida; diálisis de riñón; juegos y joguetes; niño; adolescente. 


\section{INTRODUÇÃO}

A Doença Renal Crônica (DRC) é caracterizada por perda lenta, progressiva e irreversível da função renal, que ocorre com a destruição dos néfrons, unidades funcionais dos rins, que não possuem capacidade de regeneração. Em sua fase terminal, a DRC apresenta, como uma das modalidades de tratamento, a hemodiálise, terapia de substituição da função renal realizada por meio de um sistema extracorpóreo conectado a um acesso vascular $^{1}$.

A DRC pode acometer indivíduos em diversas faixas etárias, trazendo alterações significativas em sua Qualidade de Vida Relacionada à Saúde (QVRS), considerando o seu caráter subjetivo e multidimensional. Quando afeta crianças e adolescentes, além de sua gravidade clínica, ela acomete um organismo em processo de crescimento e desenvolvimento biológico, cognitivo, social e emocional, desencadeando uma série de disfunções musculoesqueléticas, atraso puberal, desnutrição, aumentando em trinta a cinquenta vezes a taxa de mortalidade nessa faixa etária. A expectativa de vida de crianças e adolescentes de zero a 14 anos que realizam hemodiálise é de apenas 20 anos $^{2,3}$.

Diante disso, crianças e adolescentes em hemodiálise demandam uma assistência condizente com as especificidades de sua faixa etária, a qual vai além de cuidados com o domínio físico, sendo indispensável o desenvolvimento de estratégias que busquem alcançar necessidades sociais e emocionais e, assim, contribuir para o seu processo adaptativo diante das limitações impostas pela DRC e pela hemodiálise ${ }^{4}$.

A DRC exige, portanto, adaptação às mudanças e ao novo estilo de vida imposto pela patologia e pelo tratamento. Essa exigência remonta, assim, à Teoria da Adaptação de Calista Roy, que considera a atribuição da Enfermagem no processo de adaptação do indivíduo para melhor assistência à saúde e QVRS. Para Roy, o indivíduo mantém interação contínua com o ambiente, desenvolvendo respostas de acordo com os estímulos que experimenta, de modo que, quando estimulados eficientemente, desenvolvem respostas favoráveis que contribuem para a melhoria em seu estado de saúde e, consequentemente, em sua QVRS 4 .

Um tipo de estratégia que vem sendo utilizada com sucesso na aquisição e modificação de comportamentos é a gamificação. Neste tipo de estratégia de intervenção, busca-se aplicar os elementos divertidos e envolventes dos jogos em atividades comuns da vida real, gerando motivação e engajamento, contribuindo para mudanças favoráveis de comportamento ${ }^{5}$.

Sobre os "elementos de jogos" supramencionados, há diferentes concepções do que seriam em essência. Há definições em que os jogos são caracterizados pela existência de metas, regras, sistema de feedbacks e voluntariedade ${ }^{6}$ e definições estruturais em que são caracterizados a partir de dinâmicas, mecânicas e componentes ${ }^{7}$. Essas concepções são complementares e podem ser usadas para caracterizar jogos como xadrez e poker, esportes como futebol e vôlei, bem como jogos eletrônicos em todas as suas vertentes.
Assim, desenvolver uma estratégia gamificada de intervenção em saúde envolve identificar uma demanda e elencar os aspectos comportamentais relacionados a esta demanda que serão alvo da intervenção. A partir disso, definir os elementos de jogos a serem utilizados para aumentar ou reduzir os padrões de comportamento relevantes e, então, criar o protótipo da tecnologia. Neste estudo, o protótipo caracteriza-se como Tecnologia Cuidativo-Educacional (TCE), pois é um produto para a práxis profissional com convergência entre cuidado e educação ${ }^{8}$.

A produção de uma TCE envolve etapas que se iniciam pela criação de um protótipo, passam pelo processo de validação e, então, chegam à etapa de aplicação da versão final ${ }^{9}$. Em se tratando de uma TCE em formato de jogo, contudo, é recomendado que a etapa de validação seja precedida por um teste alfa que permita avaliar sua jogabilidade ao mesmo tempo em que se verifica se o protótipo atende aos objetivos para o qual foi desenvolvido, contribuindo para o levantamento de pontos que podem ser revistos e corrigidos antes da etapa de validação ${ }^{10}$

Ao partir dessas premissas, foi desenvolvido o protótipo de uma TCE gamificada em formato de um Role Playing Game (RPG) analógico, voltado a crianças e adolescentes em tratamento hemodialítico, com o objetivo principal de melhorar a sua QVRS. O jogo em questão foi denominado Nefro Hero ${ }^{\circledR 11}$ e seguiu os preceitos centrais de um RPG, que envolvem a interpretação de papéis em narrativas interativas e participativas enquanto estimulam a solução de problemas e permitem reflexões e recriações ${ }^{12}$.

O jogo retrata - de forma fantasiosa e em uma narrativa fantástica - situações vivenciadas pelas crianças e adolescentes que realizam a hemodiálise. $O$ cenário representa um mundo de fantasia composto por sete reinos que precisavam ser percorridos; cada um desses reinos está relacionado a uma condição de adaptação, a saber: Reino das Águas Turvas - não molhar o curativo do cateter; Reino do Deserto - manter restrição hídrica para evitar edema e intercorrências durante o tratamento; Reino dos Gigantes - ter déficit de crescimento, fazendo com que tenha estatura menor que a de seus pares; Reino das Vertigens - ter intercorrências e efeitos adversos da hemodiálise; Reino do Chips - manter a dieta hipossódica e restritiva; Reino do Pântano das Agulhas Negras - passar por procedimentos dolorosos; Reino do Lixo - manter cuidados de higiene e prevenção de infecção do acesso venoso. O processo de criação, que tem interface com a Teoria da Adaptação de Calista Roy, e os detalhes da jogabilidade fugiriam ao escopo deste artigo, mas podem ser encontrados na dissertação defendida pela primeira autora ${ }^{11}$.

Este artigo tem como objetivo apresentar o teste alfa do protótipo de uma tecnologia gamificada, o Nefro Hero ${ }^{\circledR}$, com foco na Qualidade de Vida Relacionada à Saúde de pacientes pediátricos em tratamento hemodialítico.

\section{MÉTODO}

Trata-se de uma pesquisa metodológica, avaliativa, com abordagem quantitativa. Foi desenvolvida em quatro etapas: primeira aplicação do inventário de QVRS DISABKIDS ${ }^{\circledR}$; nove 
sessões de jogo com os pacientes, individualmente, durante as sessões de hemodiálise; segunda aplicação do inventário; duas sessões de brainstorming.

No que tange à perspectiva avaliativa, realizada por meio do teste alfa, utilizaram-se medidas repetidas do tipo pré e pós-intervenção. Em um teste alfa de um jogo com propósito aplicado, o pesquisador deve selecionar uma ou mais variáveis que se espera que sejam afetadas pelo jogo ${ }^{10}$. Para atender a esse requisito, foi escolhido avaliar a QVRS. Delineamentos de medidas repetidas são usados, entre outros motivos, quando há um número potencialmente baixo de participantes; em estudos iniciais e para os quais ainda não se tem parâmetros claros e em estudos em que não há pretensão de generalização dos dados $^{13}$, o que é o caso deste estudo.

Com vistas a levantar os aspectos menos funcionais do jogo e que precisariam ser modificados antes da etapa de validação, foi utilizada a técnica de brainstorming em que um grupo de pessoas com perspectivas diferentes precisa chegar a um denominador comum, para determinada situação, buscando a solução de forma conjunta ${ }^{14}$.

A pesquisa foi realizada no Setor de Terapia Renal Substitutiva Pediátrica de um hospital de referência em Belém, Pará, Brasil, no período de março a maio de 2019. A escolha do local deuse por ser o único setor de hemodiálise pediátrica na região metropolitana de Belém.

Os critérios de inclusão dos participantes foram: realizar tratamento hemodialítico regular na instituição há, pelo menos, três meses e estar na faixa etária de oito a 18 anos incompletos. O critério de exclusão foi não apresentar condições cognitivas para participar do jogo. No momento da realização da pesquisa, havia 23 pacientes no setor dos quais dez não atendiam aos critérios de inclusão. Dentre os 13 restantes, dois desistiram da participação, uma foi transferida, uma recebeu alta e um veio a óbito. Assim, a amostra final foi de oito participantes, seguindo uma amostragem por conveniência em que todos os membros do universo possível foram considerados como possíveis participantes e foram incluídos todos que aceitaram participar e atendiam aos critérios previstos.

Na primeira etapa, foi aplicado o questionário de QVRS DCGM-37 do grupo DISABKIDS ${ }^{\circledR}$, que consta de três domínios distribuídos em 37 itens, organizados em uma escala Likert. As pontuações de cada domínio variam de seis (valor mínimo) a 30 (valor máximo); quanto mais alto o escore, menos afetada é a QV no respectivo domínio. O instrumento já foi traduzido e validado para o português ${ }^{15,16}$ e foi utilizado com a permissão da representante brasileira do grupo DISABKIDS ${ }^{\circledR}$. O questionário foi entregue às crianças durante uma sessão de hemodiálise e era autoaplicável, sendo preenchido pelos próprios participantes, mas com o auxílio dos pesquisadores em momentos de dificuldade de compreensão de termos ou premissas. Essa etapa ocorreu em março de 2019.

$\mathrm{Na}$ segunda etapa, realizada em nove sessões de jogo com os pacientes, individualmente, durante as sessões de hemodiálise, ocorreu a utilização do protótipo da estratégia gamificada Nefro Hero ${ }^{\circledR}$. As oito crianças e adolescentes selecionados utilizaram o jogo Nefro $\mathrm{Hero}^{\circledR} \mathrm{com}$ os seguintes itens: três imagens adesiváveis de avatares escolhidos pelas próprias crianças; 24 mapas impressos, incluindo um mapa do mundo, um da vila principal e 22 "fases" distribuídas entre os sete reinos; dois dados de dez faces; uma ficha de personagem impressa onde eram registradas suas características, evolução e progresso da história; 49 miniaturas dos vilões em Medium Density Fiberboard (MDF); lápis colorido; cubos e cilindros plásticos coloridos.

As partidas ocorreram por meio de aventuras narradas pelos pesquisadores com cada paciente, em particular, durante as sessões de hemodiálise, sendo feitas adaptações na narrativa para adequá-la às diferentes faixas etárias e particularidades de cada participante. Antes de iniciar a primeira partida, eram explicadas, para o participante, as regras do jogo, os materiais que seriam utilizados e era solicitado que escolhesse seu avatar e iniciasse o preenchimento da ficha do personagem. Os registros dessa etapa foram feitos em um diário de campo no qual eram anotadas as observações e intercorrências no decorrer das partidas. Eram agrupadas informações referentes ao comportamento dos participantes durante toda a partida, seu humor durante o período em que jogava, além de informações acerca do ambiente, como, por exemplo, ruídos ou interrupções que pudessem comprometer o interesse do participante pelo jogo. Cada partida durava, em média, 30 minutos. Essa etapa ocorreu entre os meses de abril e maio de 2019.

Na terceira etapa, após a última partida de cada participante, o inventário foi reaplicado, seguindo os mesmos parâmetros da aplicação da primeira etapa.

$\mathrm{Na}$ quarta etapa, os pesquisadores organizaram duas sessões de brainstorming com vistas a: avaliar a jogabilidade identificar dificuldades na aplicação do jogo; levantar pontos de maior confusão para as crianças, reinos, personagens e elementos menos interessantes e atrativos e outros aspectos potencialmente negativos do protótipo. Os pesquisadores pautaram-se na própria experiência enquanto narradores das aventuras e nos registros dos diários de campo. Foram elencados os pontos mencionados por todos os pesquisadores e elaborada uma lista das mudanças necessárias para a próxima versão. Uma síntese das quatro etapas pode ser vista no fluxograma a seguir (Figura 1):

Para a análise dos dados obtidos por meio das respostas ao inventário antes e após a intervenção educativa, as repostas foram organizadas em um banco de dados modelado no Microsoft Office Access ${ }^{\circledR}$ 2016. A análise estatística foi realizada nos programas GraphPad Prism $6.0^{\circledR}$ e Biostat $5.0^{\circledR}$. Foi aplicado o teste paramétrico $t$-student pareado para a correlação dos escores obtidos antes e após a intervenção. Foi utilizado o nível de significância de $5 \%$. Como se trata de um teste alfa, não se tem a pretensão de generalização dos dados, de modo que as análises não são inferenciais e sim correlacionais, buscando investigar se eventuais mudanças nos escores pós-intervenção são significativamente diferentes daquelas encontradas na pré- 

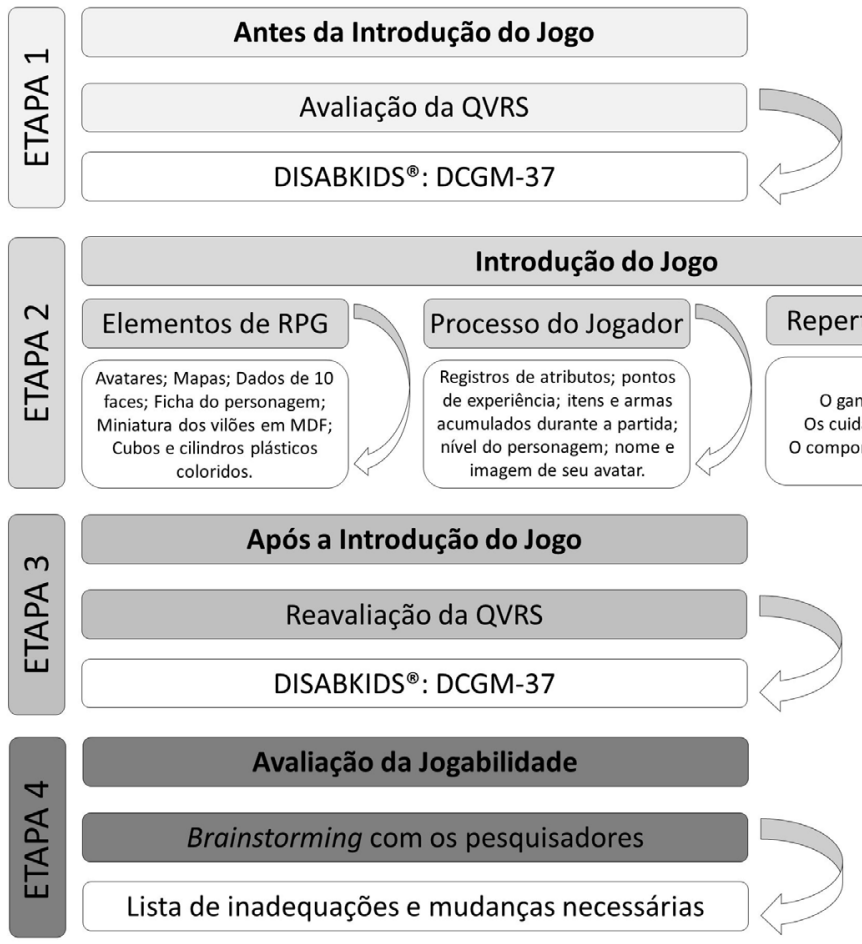

Figura 1. Fluxograma com as etapas do método.

Fonte: Elaborada pelos autores.

intervenção de modo a avaliar se essas mudanças estariam potencialmente correlacionadas à introdução do jogo.

O projeto de pesquisa foi aprovado pelo Comitê de Ética da Universidade do Estado do Pará sob o número 2.992.715 e no Comitê de Ética da Fundação Santa Casa de Misericórdia do Pará sob o número 3.159.510. Para acatar os preceitos éticos, os responsáveis pelas crianças e adolescentes assinaram o Termo de Consentimento Livre e Esclarecido (TCLE) e os menores assinaram o Termo de Assentimento Livre e Esclarecido.

\section{RESULTADOS}

O grupo de participantes foi composto de oito crianças e adolescentes, sendo cinco (62,5\%) do sexo masculino e três do sexo feminino $(37,5 \%)$, com idades entre oito e 16 anos, com média de 12,25 anos $(\mathrm{DP}= \pm 2,71)$. Quanto ao número de irmãos, cinco $(62,5 \%)$ participantes relataram ter de um a dois irmãos. Em relação à escolaridade, três (37,5\%) não estudavam e cinco $(62,5 \%)$ encontravam-se no Ensino Fundamental. Quanto ao município de residência, três $(37,5 \%)$ residiam em Belém e cinco $(62,5 \%)$ residiam em cidades do interior do Estado. Em relação ao acesso venoso, quatro (50\%) apresentam FAV e quatro (50\%), CDL. Quanto à patologia de base, quatro $(50 \%)$ tinham, como causa da DRC, a má formação congênita.

Na Tabela 1, estão apresentados os resultados relacionados aos escores de qualidade de vida antes e após a intervenção educativa gamificada. Foi verificada elevação nos escores pós- intervenção no domínio mental, com associação significativa na dimensão emocional.

No domínio mental, dimensão independência, antes da intervenção, obteve-se escore médio de 21.0 (o escore, nessa dimensão, varia de seis a 30 , sendo considerados "satisfatórios" valores mais próximos de 30). No momento pós-intervenção, o escore foi de 24.5 ( $p=0.0524)$. Na dimensão emocional, o escore médio pré-intervenção foi de 26.1 (o escore, nessa dimensão, varia de sete a 35 , sendo considerados "satisfatórios" valores mais próximos de 35 ). Após a intervenção, o escore foi de $28.8(p=0.0436)$.

No domínio social, dimensão inclusão social, antes da intervenção, o escore foi de 21.6 (o escore dessa dimensão varia de seis a 30 , sendo considerados "satisfatórios" valores próximos de 30). Após a intervenção, o escore foi de $23 \cdot 3(p=0,2308)$. $\mathrm{Na}$ dimensão exclusão social, antes da intervenção, o escore foi de 24.3 (o escore, nessa dimensão, varia de seis a 30 , sendo considerados "satisfatórios" valores mais próximos de 30 ). Após a intervenção, obteve-se escore 23.3 ( $p=0,4786)$.

No domínio físico, a dimensão limitação física apresentou escore médio pré-intervenção de 22.4 (o escore, nessa dimensão, varia de seis a 30 , sendo considerados "satisfatórios" valores mais próximos de 30 ). Após a intervenção, o escore foi de $22.9(\mathrm{p}=0,5727)$. A dimensão tratamento apresentou, na préintervenção, escore médio de 22.9 (o escore, nessa dimensão, varia de seis a 30 , sendo considerados "satisfatórios" valores 
Tabela 1. Escores de qualidade de vida dos participantes nas etapas pré e pós-intervenção educativa gamificada. Belém, Pará, Brasil, 2019. ( $\mathrm{N}=8)$

\begin{tabular}{|c|c|c|c|c|c|c|}
\hline Domínio & Dimensão & Antes & Depois & $\begin{array}{l}\text { Média das } \\
\text { diferenças }\end{array}$ & $\begin{array}{c}\text { IC 95\% } \\
\text { Médias } \\
\text { Diferenças }\end{array}$ & $\mathrm{p}$ valor ${ }^{1}$ \\
\hline Mental & Emocional & 26.1 & 28.8 & 2.6 & (0.10 a 5.15$)$ & 0.0436 \\
\hline Social & Inclusão social & 21.6 & 23.3 & 1.6 & $(-1.30$ a 4.55$)$ & 0.2308 \\
\hline \multirow{2}{*}{ Físico } & Limitação física & 22.4 & 22.9 & 0.5 & $(-1.50$ a 2.50$)$ & 0.5727 \\
\hline & Tratamento & 22.9 & 23.3 & 0.4 & $(-4.12$ a 4.89$)$ & 0.8498 \\
\hline
\end{tabular}

${ }^{(1)}$ Teste paramétrico $t$-student pareado ( $p$ valor $<0,05$ )

mais próximos de 30$)$. Após a intervenção, o escore foi de 23.3 ( $p=0,8498)$.

Em relação à avaliação da jogabilidade, os pontos negativos elencados pelos pesquisadores foram organizados em três eixos: estruturais, mecânicos e de componentes. Como problemas estruturais, foram citadas: a ausência de um manual que guiasse melhor o aplicador; a extensão da faixa etária, que demandava adaptações substanciais nas narrativas dependendo do participante e a existência de reinos onde a narrativa não mantinha o paralelo esperado com a situação de saúde/tratamento dos participantes. Os problemas relacionados às mecânicas focaram, principalmente, em elementos sem função clara, incluindo itens, personagens e atributos que não causavam impacto na narrativa ou evolução do personagem. Já em relação aos componentes, as críticas focaram a monotonia na narrativa de alguns mundos (Lixo, Chips e Pântano) e a apresentação gráfica dos mapas em geral, que não apresentavam uma padronização de tamanho e estilo, além de, muitas vezes, não acompanharem o conteúdo das narrativas.

\section{DISCUSSÃO}

Estudos sobre o perfil epidemiológico de crianças e adolescentes com DRC são escassos, entretanto, o perfil dos participantes do estudo apresenta semelhanças com outras pesquisas realizadas que também demonstraram a predominância do sexo masculino e a idade média semelhante e quanto à causa da $\mathrm{DRC}^{1-3,17}$. Uma pesquisa realizada na Bélgica revelou que $59 \%$ dos pacientes tinham, como causa da DRC, as anomalias congênitas, resultado muito semelhante ao deste estudo em que $50 \%$ dos participantes tinham a mesma patologia de base ${ }^{18}$.

Quanto à residência dos participantes, 62,5\% moram em cidades do interior do Estado, o que revela a necessidade de deslocamentos frequentes do paciente e seus familiares para as sessões de hemodiálise e para outros procedimentos necessários ao tratamento, tornando o tratamento cansativo e desgastante e $^{1-3}$.

Quanto à escolaridade dos participantes, observou-se que $37,5 \%$ deles não estavam frequentando a escola no momento da pesquisa. Isto aponta a interrupção da vida escolar, uma das implicações que o tratamento hemodialítico acarreta à vida de crianças e adolescentes, pois a rotina frequente de procedimentos a que eles são submetidos e os horários das sessões de hemodiálise os impedem de manter sua rotina normal, como, por exemplo, ir à escola ou participar de outras atividades sociais $^{1-3,17}$.

Em relação aos impactos do jogo na QVRS dos participantes, foram encontrados resultados positivos no domínio mental-que corresponde às dimensões independência e emocional -, que apresentaram os melhores escores pós-intervenção, o que condiz com o foco do jogo, que eram aspectos ligados à autopercepção e ao fortalecimento da resiliência. A dimensão emocional tem relação com as preocupações emocionais e os sentimentos. Já a independência refere-se à autonomia que o indivíduo sente em relação às suas atividades e ao seu tratamento. Os resultados encontrados nesse domínio, com associação significativa na dimensão emocional, reforçam a noção de que os indivíduos são adaptáveis e que, quando estimulados, podem revelar comportamentos favoráveis ${ }^{4,6}$.

A mudança positiva descrita acima pode ser explicada pelo efeito da gamificação, que é capaz de fazer com que o indivíduo agregue, ao contexto real, as características que o jogador apresenta quando está jogando, como otimismo e determinação, reiterando uma das funções esperadas da gamificação e dos jogos em contextos aplicados ${ }^{5-7}$.

Quanto ao domínio físico, não houve melhora significativa com a intervenção. Este domínio é composto pela dimensão limitação, que tem como foco as limitações funcionais sentidas pelos pacientes em relação à sua patologia e tratamento, e a dimensão tratamento, que aborda questões sobre o impacto emocional relacionado aos múltiplos tratamentos e procedimentos invasivos e dolorosos experimentados pelas crianças e adolescentes.

As alterações referentes ao domínio limitação eram abordadas em um dos reinos, trazendo pontos sensíveis como os efeitos sentidos durante as intercorrências da hemodiálise. Ao considerar que este domínio não foi afetado pelo jogo, os pesquisadores, durante a etapa de brainstorming, consideraram 
esta como uma das fragilidades do jogo a serem corrigidas para a próxima versão.

O jogo trazia, em sua narrativa, pontos voltados à ressignificação das experiências negativas dos participantes em relação ao seu tratamento - o que inclui melhor aceitação e resiliência frente à necessidade de uso de medicamentos injetáveis e das múltiplas punções venosas que fazem parte do tratamento. Novamente, os pesquisadores consideraram que se trata de um aspecto que precisa ser mais bem desenvolvido, incluindo narrativas mais reforçadoras em que procedimentos análogos no jogo possam trazer maiores benefícios e o desenvolvimento de aventuras mais direcionadas para essa dimensão.

Sobre o domínio social, a dimensão inclusão social aborda questões referentes às crianças e aos adolescentes em fazer parte de um grupo e ser aceito por seus pares. Já a dimensão exclusão social, que apresentou piora no escore pós-intervenção, refere-se ao estigma e à sensação de exclusão da sociedade sentidos pelos pacientes, que podem estar relacionados à presença do acesso vascular e às alterações físicas causadas pela doença. Outros estudos sobre a QVRS de crianças e adolescentes em hemodiálise revelaram que a baixa adaptação às limitações relacionadas à patologia e ao tratamento são causadas principalmente por sentimentos como vergonha $e$ discriminação, desencadeados pelas alterações físicas, como a baixa estatura e a presença do acesso vascular, que fazem com que as crianças/adolescentes se sintam diferentes de seus pares ${ }^{11,15,16}$.

Esse domínio não apresentou melhora estatisticamente significante, provavelmente pelo fato de o jogo conter poucos elementos de socialização, havendo interação apenas entre o jogador e o narrador, representado pelo pesquisador. Como as sessões de jogo acontecem durante as sessões de hemodiálise, partidas coletivas com dois ou mais jogadores não são viáveis de modo que esta fragilidade identificada no teste alfa ainda não foi solucionada.

Os diferentes mapas produzidos para o jogo deram ênfase aos desafios de adaptação das crianças e adolescentes ao tratamento. Estudos sobre as dificuldades de aceitação do tratamento hemodialítico indicaram que as principais dificuldades enfrentadas por esse público se relacionam à adesão à dieta, à restrição hídrica imposta pela doença e a ter que experienciar frequentes intervenções terapêuticas, o que os impede de desfrutar de uma rotina comum nessa fase da vida ${ }^{2,11}$.

A avaliação dos escores de QVRS permitiu avaliar o Nefro Hero $^{\circledR}$ no que tange ao seu potencial de intervir na motivação e na capacidade de resiliência de crianças e adolescentes em aderir ao tratamento da hemodiálise e, consequentemente, desenvolver respostas positivas em sua percepção de QVRS O teste alfa também foi importante para avaliar a adequação da jogabilidade e o tempo previamente definido para as partidas, pontos que não são avaliados no processo de validação.

O brainstorming levantou os pontos considerados necessários para mudança em vários aspectos do jogo relacionados à jogabilidade, revelou a necessidade de formulação de um manual de instruções, o fortalecimento de aspectos abordados nas narrativas e dessas com os mapas, a mudança do nome de vilões e os personagens não jogáveis (interpretados pelo narrador/pesquisador)

\section{CONCLUSÕES E IMPLICAÇÕES PARA A PRÁTICA}

Cada pessoa é considerada um sistema adaptativo, que desenvolve respostas mediante estímulos internos e externos. Crianças e adolescentes com DRC vivenciam mudanças em sua vida nos mais variados aspectos (ambiental, físico e social), que impõem, a essa população, alterações cotidianas que têm impacto em suas relações sociais, além dos psicológicos, pois têm que lidar com a cronicidade da doença, com o peso do tratamento e com a expectativa de um transplante renal, que envolve um processo moroso.

Diante disso, torna-se importante o desenvolvimento de tecnologias que considerem todos esses aspectos e que passem por um processo rigoroso em todas as suas etapas. A realização do teste alfa do Nefro Hero ${ }^{\circledR}$ foi importante para avaliar e elencar melhorias no protótipo antes da etapa da validação, avaliando aspectos de jogabilidade e seu potencial em desenvolver mudanças na QVRS das crianças e adolescentes que realizam hemodiálise.

A melhoria no escore emocional demonstrou o potencial da gamificação em uma possível ressignificação de aspectos relacionados ao tratamento, fortalecendo a capacidade de resiliência.

Por tratar-se de um teste alfa do protótipo de uma tecnologia, este estudo tem como limitação a não generalização dos dados. Ressalta-se que as fragilidades identificadas no teste alfa serão fortalecidas e corrigidas na nova versão da tecnologia e, posteriormente, submetidas ao processo de validação.

\section{CONTRIBUIÇÕES DOS AUTORES}

Desenho do estudo. Fernanda de Nazaré Almeida Costa. Elizabeth Teixeira. Paulo Elias Gotardelo Audebert Delage. Coleta ou produção dos dados. Fernanda de Nazaré Almeida Costa.

Análise de dados. Fernanda de Nazaré Almeida Costa Paulo Elias Gotardelo Audebert Delage. Elizabeth Teixeira. Mary Elizabeth de Santana.

Interpretação dos resultados. Fernanda de Nazaré Almeida Costa. Paulo Elias Gotardelo Audebert Delage. Elizabeth Teixeira. Marcia Helena Machado Nascimento. Mary Elizabeth de Santana.

Redação e revisão crítica do manuscrito. Fernanda de Nazaré Almeida Costa. Paulo Elias Gotardelo Audebert Delage. Mary Elizabeth de Santana. Marcia Helena Machado Nascimento. Elizabeth Teixeira.

Aprovação da versão final do artigo. Fernanda de Nazaré Almeida Costa Paulo Elias Gotardelo Audebert Delage. Mary Elizabeth de Santana. Marcia Helena Machado Nascimento. Elizabeth Teixeira. 
Responsabilidade por todos os aspectos do conteúdo e a integridade do artigo publicado. Fernanda de Nazaré Almeida Costa. Paulo Elias Gotardelo Audebert Delage. Mary Elizabeth de Santana. Marcia Helena Machado Nascimento. Elizabeth Teixeira.

\section{EDITOR ASSOCIADO}

\section{Eliane Tatsch Neves (D)}

\section{EDITOR CIENTÍFICO}

\author{
Ivone Evangelista Cabral (iD
}

\section{REFERÊNCIAS}

1. KonstantynerT, Sesso R, Camargo MF, Santis Feltran L, Koch-Nogueira PC. pediatric chronic dialysis in Brazil: epidemiology and regional inequalities. PLoS One. 2015;10(8):e0135649. http://dx.doi.org/10.1371/ journal.pone.0135649.

2. Maciel AC, Miranda JOF. Profile of children and adolescents with chronic renal failure followed up at nephrology units. J Res Fundam Care. 2013;5(3):94-103. http://dx.doi.org/10.9789/2175-5361.2013v5n3p94.

3. Lise F, Santos BP, Neutzling A, Milbrath VM, Schwartz E. Prevalência de internações e mortalidade infantile por insuficiência renal no brasil. Rev Enferm UFPE on line [Internet]. 2017; [citado 2021 fev 4];11(8):3295-302 Disponível em: https://periodicos.ufpe.br/revistas/revistaenfermagem/ article/view/110196/22088

4. George JB. Teorias de enfermagem: os fundamentos a prática profissional. 4. ed. Porto Alegre: Artmed; 2000. $355 p$

5. Bozkurt A, Durak G. A systematic review of gamification research: in pursuit of homo ludens. Int J Game-Based Learn. 2018;8(3):15-33. http://dx.doi.org/10.4018/IJGBL.2018070102.

6. McGonigal J. A realidade em jogo: porque os games nos tornam melhores e como eles podem mudar o mundo. 1. ed. Rio de Janeiro: Best Seller; 2012.

7. Dichev C, Dicheva D. Gamifying education: what is known, what is believed and what remains uncertain: a critical review. Technol High Educ. 2017;14:9. http://dx.doi.org/10.1186/s41239-017-0042-5.

8. Salbego C, Nietsche EA, Teixeira E, Girardon-Perlini NMO, Wild CF, Ilha S. Care-educational technologies: an emerging concept of the praxis of nurses in a hospital context. Rev Bras Enferm. 2018;71(Supl. 6):2666-74. http://dx.doi.org/10.1590/0034-7167-2017-0753.

9. Teixeira E, Nascimento MHM. Pesquisa metodológica: perspectivas operacionais e densidades participativas. In: Teixeira E, organizador. Desenvolvimento de tecnologias cuidativo-educacionais. Porto Alegre: Moriá; 2020. p. 51-62. (vol. II).

10. Sperhacke SL. Aprendizagem de métodos de design: estudo baseado na construção e validação de jogo de tabuleiro [tese]. Porto Alegre: Programa de Pós-graduação em Design, Universidade Federal do Rio Grande do Sul; 2019 [citado 2021 fev 4]. Disponível em: https://www. lume.ufrgs.br/handle/10183/198071

11. Costa FNA. Qualidade de vida relacionada a saúde de crianças e adolescentes em hemodiálise: produção e testagem de uma estratégia gamificada [dissertação]. Belém (PA): Escola de Enfermagem Magalhães Barata, Universidade do Estado do Pará; 2019 [citado 2021 fev 4]. Disponível em: https://drive.google.com/file/d/1KQPSnqh0j6Lc1Wc9 Hts3P5dh4pKa3D8e/view

12. Soares AN, Gazzinelli MF, Souza V, Araújo LHL. The Role Playing Game (RPG) as a pedagogical strategy in the training of the nurse: an experience report on the creation of a game. Texto Contexto Enferm. 2015;24(2):600-8. http://dx.doi.org/10.1590/0104-07072015001072014.

13. Kazdin AE. Single-case research designs: methods for clinical and applied settings. 2nd ed. Oxford: Oxford University Press; 2010.

14. Granado GCS. Brainstorming e a aplicação do modelo clássico. Rev Cient Multidiscip Núcleo Conhecimento. 2020;18(10):5-20. http://dx.doi. org/10.32749/nucleodoconhecimento.com.br/engenharia-de-producao/ brainstorming

15. Fegadolli C, Reis RA, Martins STA, Bullinger M, Santos CB. Adaptação do módulo genérico DISABKIDS $₫$ para crianças e adolescentes com condições crônicas. Rev Bras Saúde Matern Infant [Internet]. 2010; [citado 2021 fev 4];10(1):95-105. Disponível em: https://www.scielo. br/j/rbsmi/a/XyQJ9q8bkxGjMGfLsH68xWw/?format=pdf\&lang=pt

16. Nunes SPH.Validation of the DISABKIDS $®-37$ instrument for Brazilian children and adolescents with chronic conditions [tese]. Ribeirão Preto: Escola de Enfermagem de Ribeirão Preto, Universidade de São Paulo; 2014 . https://doi.org/10.11606/T.22.2014.tde-30032015-185317.

17. Santos RP, Rocha DLB, Koch VHK. Components clínico-epidemiológicos de crianças e adolescentes em hemodiálise. Cogitare Enferm. 2014;19(20):362-7. http://dx.doi.org/10.5380/ce.v19i2.33924.

18. Hiep TTM, Ismaili K, Collart F, Damme-Lombaerts RV, Godefroid N, Ghuysen MS et al. Clinical caracteristics and outcomes children with estage 3-5 chronic kidney disease. Pediatr Nephrol. 2010;25(5). http:// dx.doi.org/10.1007/s00467-009-1424-2. 